\title{
Endoscopic ultrasound-guided treatment of gastric varices with coil embolization and absorbable hemostatic gelatin sponge: a novel alternative to cyanoacrylate
}

(๑) $\circledast \ominus$

\author{
Authors \\ Institutions \\ 1 Division of Gastroenterology, Hepatology and \\ Endoscopy. Brigham and Women's Hospital, Boston, \\ Massachusetts, United States \\ 2 Department of Internal Medicine. Massachusetts \\ General Hospital. Boston, Massachusetts, United States \\ 3 Harvard Medical School, Boston, Massachusetts, United \\ States
}

Ahmad Najdat Bazarbashi, ${ }^{1,3}$, Thomas J. Wang ${ }^{2,3}$, Christopher C. Thompson ${ }^{1,3}$, Marvin Ryou ${ }^{1,3}$ bleeding gastric varices. submitted 17.4.2019

accepted after revision 15.7 .2019

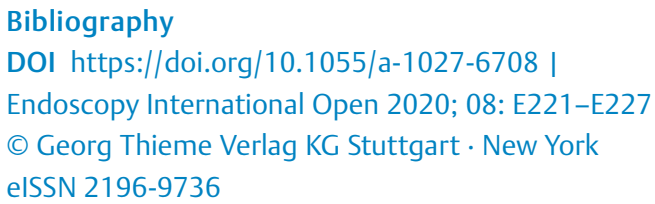

Corresponding author

Marvin Ryou, Brigham \& Women's Hospital, 45 Francis St., ASB-II, Boston, MA 02115

Fax: +1-617-566-0338

mryou@bwh.harvard.edu

\section{ABSTRACT}

Background and study aims Bleeding from gastric varices (GV) carries high morbidity and mortality. Current endoscopic therapies are premised on cyanoacrylate injection which is technically challenging and carries risk of em- bolization. We present a case series of endoscopic ultrasound (EUS)-guided coil injection in combination with hemostatic absorbable gelatin sponge (AGS) for treatment of

Patients and methods This was a retrospective review of EUS-guided coil injection for bleeding GV since November 2017. After EUS-guided needle puncture, hemostatic coils were serially injected until significant reduction of Doppler flow. Under fluoroscopic guidance, test contrast was injected to confirm absence of run-off, at which time AGS, converted into a liquid slurry, was injected as hemostatic reinforcement.

Results Ten consecutive patients underwent EUS-guided coil embolization reinforced by AGS. Technical success, defined as uncomplicated injection of coils and sponge was achieved in $100 \%$ (10/10). Mean follow-up was 6 months 73-397 days; No patients rebled or required reintervention on GV. The complication rate was $10 \%(1 / 10$; severe abdominal pain without radiographic findings); otherwise, there were no cases of systemic embolization. Nine of 10 patients (90\%) had follow-up EUS (mean 80 days); $100 \%$ (9/9) revealed near-obliteration of GV.

Conclusion EUS-guided coil embolization in combination with hemostatic AGS is a novel method for management of bleeding GV with high clinical and technical success rates, low risk for complications and favorable safety profile when compared to cyanoacrylate. This technique theoretically minimizes embolic complications and need for re-intervention. Further studies are required to compare this modality.

\section{Introduction}

Variceal bleeding is a common presentation of gastrointestinal bleeding (GIB) and is associated with significant morbidity and mortality. Gastroesophageal varices are present in at least half of patients diagnosed with cirrhosis [1]. Gastric variceal (GV) bleeding occurs less frequently compared to esophageal variceal bleeding, but is associated with more severe bleeding and higher mortality rates [2]. While portal hypertension remains the most common cause of gastric varices (GV) in the United
States, splenic vein thrombosis (SVT) presents another common cause seen in clinical practice.

Despite being associated with more severe bleeding and higher morbidity and mortality rates, GV bleeding is associated with fewer well-defined treatment options. Historically, endoscopic therapy for GV has been premised on the injection of acrylate polymers, such as histoacryl and cyanoacrylate, which has yielded promising results [3-7]. However, despite effective bleeding cessation, injection of these synthetic polymers can be difficult to perform in inexperienced hands and has been 
associated with complications, such as systemic embolization, failed withdrawal of needle from variceal nest after injection and deep ulceration resulting in re-bleeding [8-11]. To mitigate against these complications, cyanoacrylate has been used as adjunctive therapy following hemostatic coils delivered intravascularly under endoscopic ultrasound (EUS) guidance [12, 13]. Theoretically coils provide scaffolding for localized glue polymerization, however adverse events including rebleeding and embolization are still seen with combination therapy [13, 14].

Absorbable gelatin sponge [AGS] has been widely used as a hemostatic agent in surgery and interventional radiology (IR), including as an intravascular hemostatic adjunct in lieu of sclerosants and synthetic glues for the treatment of GV (CARTO) [15]; however, use of absorbable gelatin sponge has not been previously described as an endoscopic treatment. AGS is a purified collagen that forms a plug by absorbing up to 45 times its volume in blood [16], and has been observed to liquefy after approximately one week [17]. Unlike cyanoacrylate, it has not been associated with post-injection ulcerations and is not known to damage scopes. In light of these theoretical benefits of absorbable gelatin sponge, we herein present a case series of a novel endoscopic intervention for the treatment of gastric varices involving EUS guided hemostatic coil embolization in combination with absorbable gelatin sponge injection.

\section{Patients and methods}

We conducted a retrospective review of prospectively collected data on ten consecutive patients with active or recent gastric variceal bleeding who had undergone EUS-guided coil embolization between October 2017 and November 2018. Institutional review board (IRB) approval was obtained from our hospital. Detailed written consent was obtained from each patient or healthcare proxy prior to intervention.

Baseline demographics and procedure characteristics were obtained for each patient from available medical records, endoscopy reports and archived video records. Patient data collected included Charlson's morbidity score, etiology of GV, etiology of liver disease if present, severity of underlying liver disease as per Model for End Stage of Liver Disease [MELD] and Child-Pugh Score, GV type based on Sarin's classification [18] and endosonographic size of GV. Procedure related data collected included number and length of coils injected, use of absorbable gelatin sponge, complications including presence of post procedural bleeding and result of follow-up. Means with standard deviations and ratios/percentages were calculated for baseline patient and procedure related data.

\section{Procedural details}

All procedures were performed at our medical center's endoscopy unit. Each case was video recorded and archived. Absorbable Gelatin Sponge (either Gelfoam [Pfizer, New York, NY, USA] or Surgiflo [Johnson \& Johnson Wound Management, Somerville, New Jersey, United States] was converted into a liquid slurry by mixing the sponge with a 1:1 solution of saline and contrast (Omnipaque, GE Healthcare, Chicago, Illinois, United
States] under sterile technique. A total of 3-5cc of absorbable gelatin sponge was prepared for each case ( $\triangleright$ Fig. $1 \mathbf{a}-\mathbf{e})$.

All procedures were performed under general anesthesia with each patient positioned supine initially. All patients underwent upper endoscopy (GIF-HQ190, Olympus America Inc, Center Valley, Pennsylvania, United States) prior to EUS to evaluate for active bleeding and further characterize the GV to be intervened upon. The upper endoscope was exchanged for a linear echoendoscope (GF-UCT180), Olympus America Inc, Center Valley, PA] to delineate GV anatomy and assess for shunts. Saline was instilled into the gastric lumen with the patient shifted to left lateral decubitus to fill the gastric fundus and allow optimal visualization of GV nests and associated shunts. Once the target GV nest was identified, transesophageal needle puncture, using a 19-G needle (Expect needle, Boston Scientific, Natick, Massachusetts, United States) in 9/10 patients, was performed. A 22-G needle (Expect needle, Boston Scientific, Natick, Massachusetts, United States) was used in one patient due to the presence of small gastric varices on EUS requiring a smaller-gauge needle. GV puncture was performed under EUS guidance. After needle puncture, multiple cylindrical-shaped coils (Nester Embolization Coils, Cook Medical, Bloomington, Indiana, United States);( 0.035" if using $19 \mathrm{G}$ needle; 0.018 " if using $22 \mathrm{G}$ needle with varying lengths of $7 \mathrm{~cm}$, $14 \mathrm{~cm}$ and $21 \mathrm{~cm}$ and diameter range fo $12-20 \mathrm{~mm}$ ) were injected into the GV nest, under EUS and fluoroscopic guidance. Needle puncture was performed with the intent of coil anchoring, especially for the first coil injection. The needle was advanced into the distal-most compartment near the feeder vessel, which was then "packed" with as much coil as possible. The needle was withdrawn across a vessel wall into the next compartment and the coil packing was resumed. The reason for this technique was two-fold: (1) to maximize length of coil inserted, particularly with the first injection, and (2) to anchor the first coil. Finally, after coil injection, an average $1-3 \mathrm{cc}$ of AGS (Gelfoam or Surgiflo) in combination with test contrast, was injected under fluoroscopic guidance to reinforce hemostasis except in once case due to the presence of persistent shunt post coil injection increasing the risk of systemic embolization ( $\triangleright$ Fig.2-e). Technical success was defined as uncomplicated injection of coils and AGS with concomitant reduction or cessation of Doppler flow within the gastric varix.

All patients underwent follow up endoscopic ultrasound within 1 to 3 months to confirm decompression of GV. Successful GV decompression was defined as greater than 75\% GV size reduction endoscopically and/or greater than $75 \%$ doppler flow reduction sonographically.

\section{Results}

Ten consecutive patients underwent EUS-guided coil embolization for treatment of gastric varices. Demographics and patient characteristics are summarized in - Table 1. Mean age was 64 years, $60 \%$ patients were female. The most common cause of gastric varices was alcohol-related cirrhosis and portal hypertension (50\%). One patient had non-cirrhotic related gastric varices secondary to splenic vein thrombosis. Notably, active 

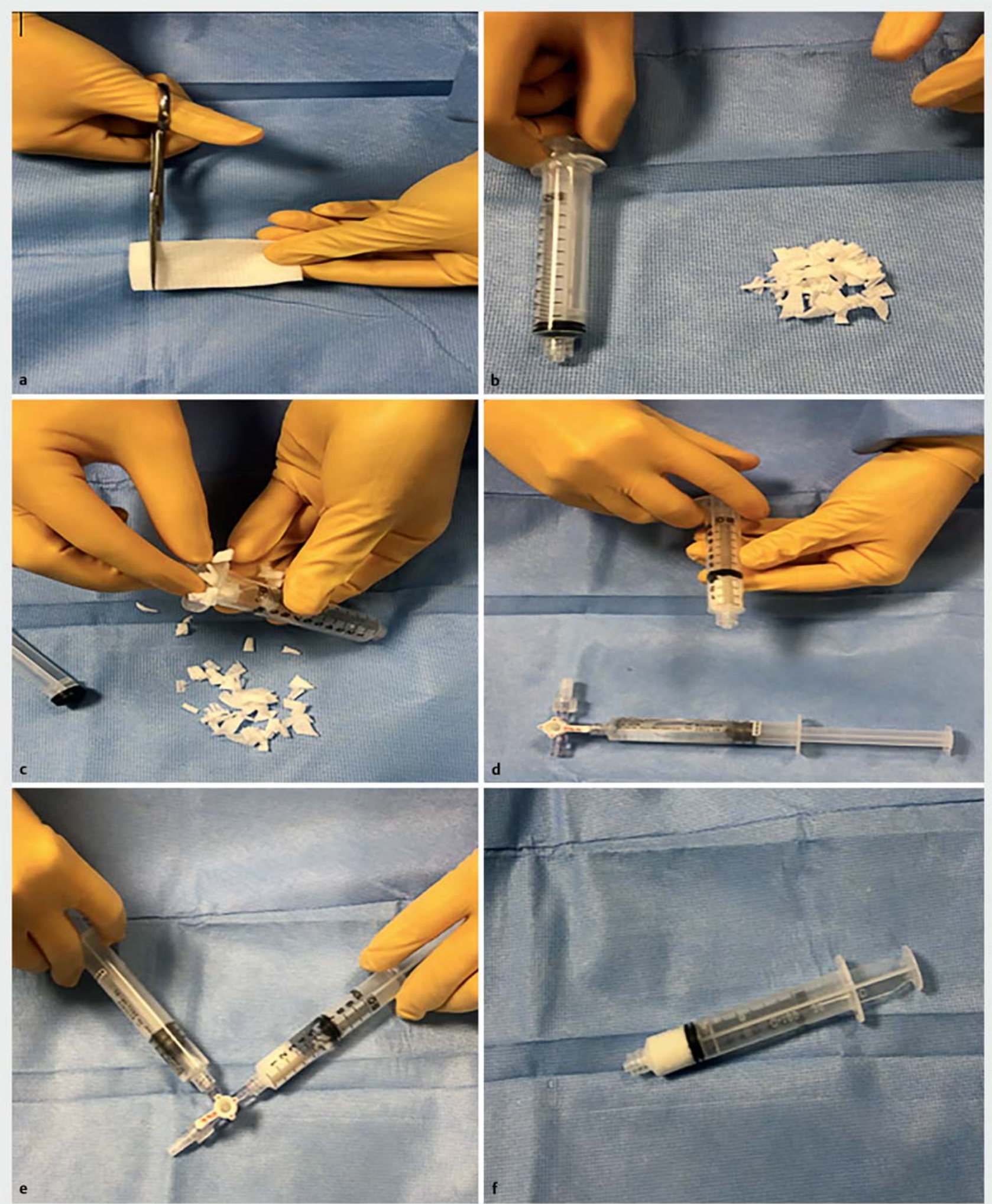

- Fig. 1 Absorbable gelatin sponge preparation and conversion to a liquid slurry. a, b Absorbable gelatin sponge strips are cut into small fragments. c,d Absorbable gelatin sponge fragments are packed into a 10-cc syringe. e, $\mathbf{f} A$ three-way stop-cock is used to mix $5 \mathrm{cc}$ of saline with the absorbable gelatin sponge fragments until the mixture is converted into a liquid slurry. 

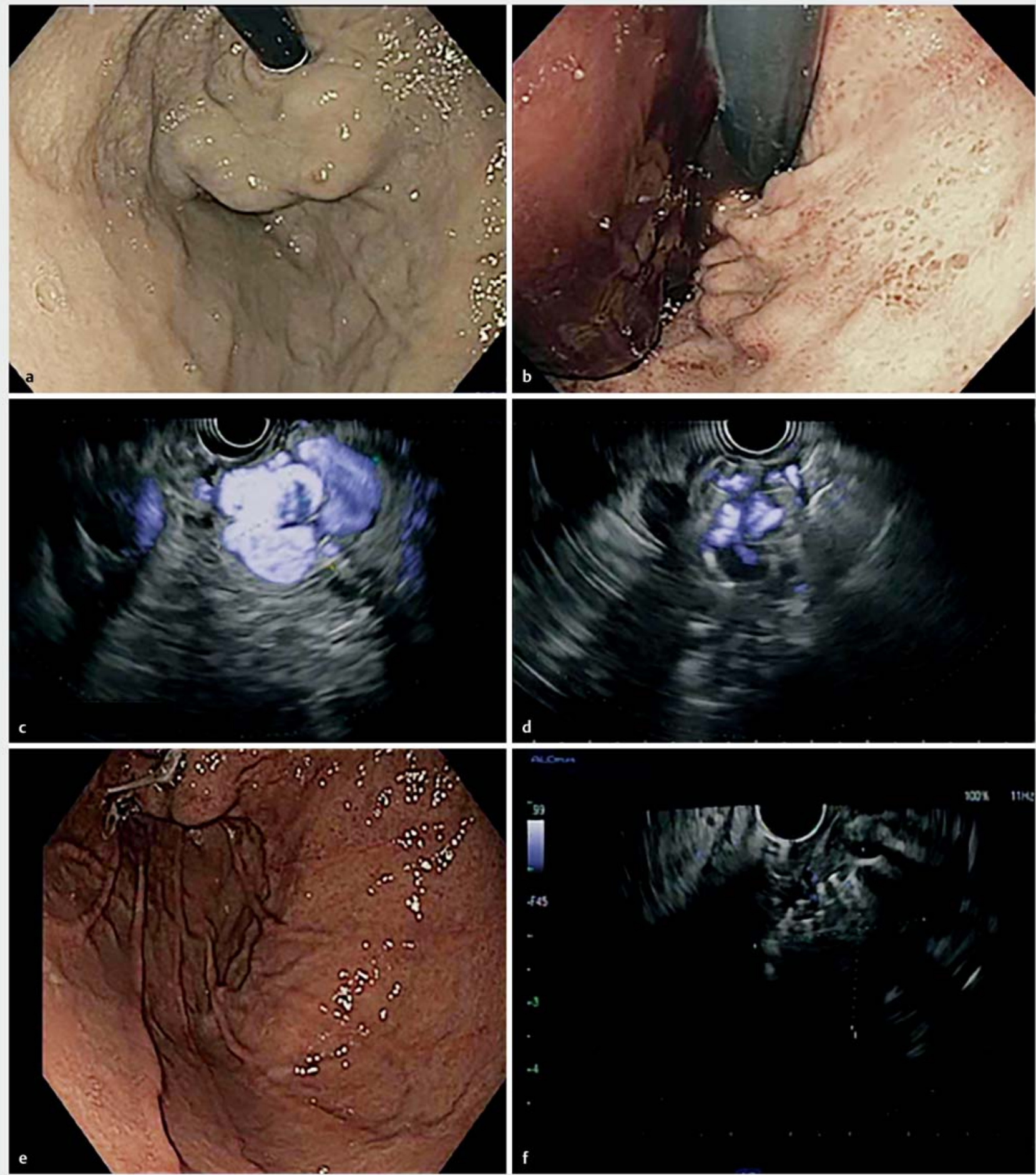

- Fig. 2 Treatment of gastric varices using EUS-guided coil and AGS injection. a Retroflexed view of fundal isolated gastric varices (IGV1) prior to episode of bleeding. $\mathbf{b}$ Large clot occupying stomach, obscuring adequate endoscopic visualization of gastric varices. $\mathbf{c}$ Endosonographic image with doppler flow of IGV1 prior to EUS-guided coil embolization and Gelfoam injection. $\mathbf{d}$ Endosonographic image after EUS-guided embolization and Gelfoam injection confirming reduced Doppler flow. e Six-week follow-up with repeat EGD revealing collapsed GV, with benign coil tip extrusion. $\mathbf{f}$ Six-week follow-up with repeat EUS revealing complete obliteration of GV without residual Doppler flow.

or recent hemorrhage was present in nine of 10 patients (90\%). Mean pre-procedure blood transfusion requirement was two units of packed red blood cells. Mean Child Pugh score was
Class B with mean MELD of 14 (SD 5.6). $70 \%$ (7/10) had isolated gastric varices (IGV1). Mean GV size was 24 (SD 14.6) mm. 
- Table 1 Patient baseline characteristics.

\begin{tabular}{|c|c|c|c|c|c|c|c|c|}
\hline & Age (years) & Sex & $\mathrm{CCl}$ & Etiology of GV & CTP Class & MELD & Type of GV & Size of GV $(\mathrm{mm})$ \\
\hline Case 1 & 51 & $F$ & 5 & Etoh Cirrhosis & C & 17 & GOV2 & 12 \\
\hline Case 2 & 71 & $\mathrm{~F}$ & 7 & Etoh Cirrhosis & B & 8 & IGV1 & 39 \\
\hline Case 3 & 56 & $F$ & 4 & Etoh Cirrhosis & A & 7 & IGV1 & 22 \\
\hline Case 4 & 73 & M & 6 & Etoh Cirrhosis & B & 13 & IGV1 & 18 \\
\hline Case 5 & 71 & $\mathrm{~F}$ & 7 & NAFLD Cirrhosis & B & 15 & IGV1 & 12 \\
\hline Case 6 & 49 & M & 3 & Etoh Cirrhosis & C & 21 & GOV2 & 15 \\
\hline Case 7 & 58 & $M$ & 1 & $\begin{array}{l}\text { Pancreatitis with } \\
\text { SVT }\end{array}$ & $\mathrm{N} / \mathrm{A}$ & $\mathrm{N} / \mathrm{A}$ & IGV1 & 23 \\
\hline Case 8 & 84 & $\mathrm{~F}$ & 8 & NAFLD Cirrhosis & A & 21 & GOV2 & 36 \\
\hline Case 9 & 71 & $\mathrm{~F}$ & 10 & NAFLD Cirrhosis & A & 8 & IGV1 & 35 \\
\hline Case 10 & 55 & M & 5 & A1AT & B & 19 & IGV1 & 50 \\
\hline Mean \pm SD & $64 \pm 11.5$ & & $6 \pm 2.6$ & & B & $14 \pm 5.6$ & & $24 \pm 14.6$ \\
\hline
\end{tabular}

CC, Charlson Comorbidity Index; CTP, Child Pugh score; NAFLD, non-alcoholic fatty liver disease; SVT, splenic vein thrombosis; A1AT, alpha 1 antitrypsin deficiency

- Table 2 Procedure characteristics.

\begin{tabular}{|c|c|c|c|c|c|c|}
\hline & $\begin{array}{l}\text { Number } \\
\text { of coils }\end{array}$ & $\begin{array}{l}\text { Total length } \\
\text { of coil (cm) }\end{array}$ & $\begin{array}{l}\text { Absorbable gela- } \\
\text { tin sponge use }\end{array}$ & $\begin{array}{l}\text { Absorbable gelatin } \\
\text { sponge volume (mL) }\end{array}$ & $\begin{array}{l}\text { Follow-up } \\
\text { time (days) }\end{array}$ & $\begin{array}{l}\text { Time to repeat } \\
\text { EUS (days) }\end{array}$ \\
\hline Case 1 & 8 & 77 & Yes & 3 & 397 & 125 \\
\hline Case 2 & 10 & 174 & Yes & 2 & 264 & 92 \\
\hline Case 3 & 11 & 147 & Yes & 3 & 299 & 33 \\
\hline Case 4 & 8 & 112 & Yes & 2 & 236 & 110 \\
\hline Case 5 & 8 & 134 & No & N/A & 171 & 85 \\
\hline Case 6 & 12 & 204 & Yes & 3 & 120 & 120 \\
\hline Case 7 & 3 & 42 & Yes & 2 & 124 & 49 \\
\hline Case 8 & 5 & 100 & Yes & 3 & $N / A^{*}$ & $N / A^{*}$ \\
\hline Case 9 & 6 & 120 & Yes & 2 & 82 & 50 \\
\hline Case 10 & 5 & 77 & Yes & 4 & 73 & 60 \\
\hline Mean \pm SD & $8 \pm 2.9$ & $119 \pm 48.4$ & & $2.5 \pm 0.7$ & $196 \pm 110$ & $80 \pm 33$ \\
\hline
\end{tabular}

Procedure characteristics are summarized in > Table 2. Technical success, defined as as successful EUS-guided injection of hemostatic coils with or without AGS into gastric variceal nests as confirmed by endoscopic ultrasonography and fluoroscopy was $100 \%$ (10/10 cases) for EUS-guided coil embolization with absorbable gelatin sponge co-injected in 9/10 cases. A mean of eight coils (SD 2.9) were deployed per case with an average total coil length of $119 \mathrm{~cm}($ SD $48 \mathrm{~cm})$. AGS (Gelfoam or Surgiflo) was injected as liquid slurry and was used for hemostatic reinforcement after coil embolization in all cases except for one due to persistent flow involving gastrorenal shunt increasing risk for systemic embolization.
There were no intraprocedural complications (e. g. bleeding or new hemodynamic instability) observed. Hemoglobin stabilized in all patients with immediate cessation of transfusion requirements. Two patients (20\%) developed transient low-grade fever within 24 hours, which resolved without intervention. One patient, who had a history of chronic abdominal pain, developed severe post-procedure pain $(10 \%)$ with reassuring CT and MRI scans and her pain eventually resolved with supportive care. One patient died 2 days post-intervention from non-procedure-related causes as she was transitioned to comfort care due to multiple comorbidities (elderly patient with acute lymphoma in blast crisis and acute renal failure). 
Mean clinical follow-up was 196 days (SD 110 days). All patients had follow-up endoscopy after a mean 80 days (SD 33 days) from index treatment at which time EUS confirmed significant decompression or obliteration of gastric varices with absent or significantly reduced Doppler flow. There was no evidence of AGS extrusion or ulcer formation. Of note, one patient $(10 \%)$ rebled with small-volume coffee ground emesis after 5 months; however, on upper endoscopy he was found to have erosive esophagitis with superficial ulcers as the bleeding source and his GV appeared decompressed on EUS.

\section{Discussion}

Gastric variceal bleeding is associated with significant morbidity and mortality with fewer well-defined management options compared to esophageal variceal bleeding [19]. Historically, endoscopic therapy for GV has relied on intravenous injection of acrylate polymers often used in conjunction with lipiodol, a radio-opaque ionized oil which slows polymerization of these polymers allowing more time for injection [19]. Cyanoacrylate injection has been associated with multiple complications [812], most notably systemic embolization (e.g. pulmonary embolism) in up to $2 \%$ to $3 \%$ of cases, and sclerosant or glue extrusion which can occur within 1 to 3 months and can lead to recurrent variceal bleeding [19-21]. Other complications include fevers, sepsis, and gastric ulcer formation. Furthermore, particularly when used in in-experienced hands, cyanoacrylate can freeze within the injection needle, can cause significant damage to endoscopes, and can unroof varices if polymerized onto the needle tip. While combining coils with CYA to mitigate again possible complications has revealed high technical and clinical success rates, adverse events have still been observed. In the largest series to day on combination therapy with coils and glue by Bhat et al, a total of 20 of 125 patients (16\%) had post-treatment bleeding from GV and non GV causes, with 10 of 125 patients (8.0\%) occuring from GV causes [13].

This case series utilized AGS, such as Gelfoam, as an alternative to cyanoacrylate for adjunctive therapy with EUS-delivered coils. Notably, Gelfoam - a purified collagen that absorbs 45 times its volume in blood [15] is already used as a hemostatic agent and has been used for decades in surgery and interventional radiology, and there is even a precedent for combination AGS/coil treatment of GV (coil-assisted retrograde transvenous obliteration or CARTO) [15]. Adjunctive AGS may be superior to adjunctive cyanoacrylate for several reasons: it does not damage the scope, its viscosity can be adjusted to avoid premature polymerization within the needle lumen, liquefies within 1 week [17] and its current use for hemostasis has been well establish in IR and surgery literature. This series is the first to report use of AGS injection to treat GV through endoscopic means.

Overall, in this case series of patients with gastric varices that were actively bleeding or had recently bled, endoscopic therapy using combination coils and AGS appeared safe and effective. Technical success, defined as intravascular delivery of both coils and/or AGS with concomitant cessation or significant reduction of Doppler flow in the gastric variceal nest, was achieved in $100 \%$ of cases. Complications typically associated with cyanoacrylate injection (e.g. embolic complications, post injection ulcers, etc) did not occur in this cohort. There was immediate cessation of transfusion requirements post-procedure. After a mean follow-up period of 6 months, no patients rebled from recurrent gastric or esophageal variceal bleeding or required re-intervention. Follow-up EUS examination confirmed GV decompression in all cases. Adverse events were minor and consisted of one patient (10\%) with post-procedure abdominal pain that was treated conservatively, although that patient had preexisting abdominal pain prior to her endoscopic treatment.

Several aspects of the endoscopic technique should be highlighted. First, as previously described $[22,23]$, instilling fluid into the stomach and rolling the patient leftward to induce pooling in the fundus helps to isolate the culprit gastric variceal nest on EUS, as often the diffuse nature of collateral vessels can be initially confusing. Second, transesophageal puncture of the GV nest is ergonomically advantageous, as the EUS scope is in a relatively straight configuration. Third we injected very long coils (usually 14 or $21 \mathrm{~cm}$ in length), working in the distal-most vascular compartment of the serpiginous nest with the attempt to target the feeder vessel if possible. Often, the needle was withdrawn across a vessel wall to resume coiling in a proximal compartment, essentially anchoring the coil across that vessel wall. (This concept of coil anchoring across vessel walls does not exist in interventional radiology given current practice of coil extrusion within a single compartment.) It is likely that this technique allowed for deloyment of a larger number of coils with total lengths exceeding $100 \mathrm{~cm}$ compared to published literature. Fourth, unlike other published studies on coil injection for GV, we utilized a higher than average number of coils (mean of 8 coils per patient). Fifith, concomitant fluoroscopy was used to guard against embolic complications. When Doppler interrogation indicated significant reduction of venous flow following coil deployment, a test contrast injection was performed. If contrast was locally retained, AGS was subsequently injected, effectively ceasing all flow. A mean of 2.5 cc of gelfoam was injected, with the goal of 2 to 3 cc per case, a similar fashion to the volume of cyanoacrylate published in other studies. Case \#5 was our only case where AGS was not used due to persistent run-off of test contrast; this particular case underscores fluoroscopy as an important procedural adjunct as its absence may have otherwise led to AGS embolic complications.

Limitations of this case series include its retrospective, noncomparative design and overall small numbers. Additionally, given these small numbers, questions regarding technique optimization (e.g. How many coils? Which coils? How much Gelfoam?) cannot be answered at this time. Finally, it is difficult to discern the relative hemostatic contribution of AGS compared to the high number of coils injected per patient in this series. However, we do highlight the AGS as an acceptable and possibly safer alternative to cynoacrylate. 


\section{Conclusion}

In conclusion, EUS-guided therapy combining coils and absorbable gelatin sponge is a novel method for management of bleeding gastric varices and appears to have high technical and clinical success rates. Unlike cyanoacrylate, AGS used as a hemostatic adjunct appears to have a favorable safety profile. Results of this combination technique suggest minimization of embolic complications and the need for re-intervention. Larger studies are required to optimize technique as well as to elucidate its potential place relative to other treatment options. Future studies should compare this modality to standard endoscopic therapies in current practice and compare the use of coils with and without adjunctive AGS.

\section{Competing interests}

Ahmad Najdat Bazarbashi: None

Thomas J Wang: None

Marvin Ryou: Consultant for Medtronic, Boston Scientific and Olympus America

Christopher C Thompson: Apollo Endosurgery, Consultant for Boston Scientific, Olympus America, Medtronic, Fractyl, GI Dynamics, USGI Medical

\section{References}

[1] Garcia-Tsao G, Bosch J. Management of varices and variceal hemorrhage in cirrhosis. N Engl J Med 2010; 362: 823-832

[2] Wani ZA, Bhat RA, Bhadoria AS et al. Gastric varices: Classification, endoscopic and ultrasonographic management. J Res Med Sci 2015; 20: $1200-1207$

[3] Soehendra N, Nam VC, Grimm H et al. Endosopic obliteration of large esophagogastric varices with bucrylate. Endoscopy 1986; 18: 25-26

[4] Ramond M], Valla D, Gotlib JP et al. Endoscopic obturationof esophagogastric varices with bucrylate. I. Clinical study of 49 patients. Gastroenterol Clin Biol 1986; 10: 575-579

[5] Iwase H, Maeda O, Shimada M et al. Endoscopic ablation with cyanoacrylate glue for isolated gastric variceal bleeding. Gastrointest Endosc 2001; 53: 585-592

[6] Sarin SK, Jain AK, Jain M et al. A randomized controlled trial of cyanoacrylate versus alcohol injection in patients with isolated fundic varices. Am J Gastroenterol 2002; 97: 1010-1015

[7] Cheng LF, Wang ZQ, Li CZ et al. Treatment of gastric varices by endoscopic sclerotherapy using butyl cyanoacrylate: 10 years' experience of 635 cases. Chin Med J (Engl) 2007; 120: 2081-2085
[8] Fry LC, Neumann H, Olano C et al. Efficacy, complications and clinical outcomes of endoscopic sclerotherapy with $\mathrm{N}$-butyl-2-cyanoacrylate for bleeding gastric varices. Dig Dis 2008; 26: 300-303

[9] Dhiman RK, Chawla Y, Taneja S et al. Endoscopic sclerotherapy of gastric variceal bleeding with N-butyl-2-cyanoacrylate. J Clin Gastroenterol 2002; 35: 222-227

[10] Kok K, Bond RP, Duncan IC et al. Distal embolization and local vessel wall ulceration after gastric variceal obliteration with N-butyl-2-cyanoacrylate: a case report and review of the literature. Endoscopy 2004; 36: 442-446

[11] Rickman OB, Utz JP, Aughenbaugh GL et al. Pulmonary embolization of 2-octyl cyanoacrylate after endoscopic injection therapy for gastric variceal bleeding. Mayo Clin Proc 2004; 79: 1455-1458

[12] Robles-Medranda C, Valero M et al. Endoscopic-ultrasound guided coil and cyanoacrylate embolization for gastric varices and the roles of endoscopic Doppler and endosonographic varicealography in vascular targeting. Dig Endosc 2019; 31: 283-290

[13] Bhat YM, Weilert F, Fredrick RT et al. EUS-guided treatment of gastric fundal varices with combined injection of coils and cyanoacrylateglue: a large U.S. experience over 6 years (with video). Gastrointest Endosc 2016; 83: 1164-1172

[14] Lôbo MRA, Chaves DM, DE Moura DTH et al. Safety and efficacy of EUS-guided coil plus cyanoacrylate versus conventional cyanoacrylate technique in the treatment of gastric varices: a randomized controlled trial. Arq Gastroenterol 2019: doi:10.1590/S00042803.201900000-08 [E-pub ahead of print]

[15] Lee EW, Saab S, Gomes AS et al. Coil-assisted retrograde transvenous obliteration (CARTO) for the treatment of portal hypertensive variceal bleeding: preliminary results. Clin Translat Gastroenterol 2014; 5: e61

[16] Council on Pharmacy and Chemistry. Absorbable Gelatin sponge new and nonofficial remedies. JAMA 1947; 135: 921

[17] Jenkins HP, Senz EH, Owen $\mathrm{H}$ et al. Present status of gelatin sponge for control of hemorrhage. JAMA 1946; 132: 614-619

[18] Sarin SK, Lahoti D, Saxena S et al. Prevalence, classification and natural history of gastric varices: A long-term follow-up study in 568 portal hypertension patients. Hepatology 1992; 16: 1343-1349

[19] Seewald S, Leong T, Imazu $\mathrm{H}$ et al. A standardized injection technique and regimen ensures success and safety of $\mathrm{N}$-butyl-2-cyanoacrylate injection for the treatment of gastric fundal varices. Gastointest Endosc 2008; 68: 447-454

[20] Wang YM, Cheng LF, Li N et al. Study of glue extrusion after endoscopic N-butyl-2-cyanoacrylate injection on gastric vari- ceal bleeding. World J Gastroenterol 2009; 15: 4945-4951

[21] Joo HS, Jang JY, Eun SH et al. Long-term results of endo- scopic histoacryl (N-butyl-2-cyanoacrylate) injection for treat- ment of gastric varices: a 10-year experience. Korean J Gastroenterol 2007; 49: 320326

[22] Ge PS, Bazarbashi AN, Thompson CC et al. Successful EUS-guided treatment of gastric varices with coil embolization and injection of absorbable gelatin sponge. VideoGIE 2018; 4: 154-156

[23] Bazarbashi AN, Ryou M. EUS-guided coil injection therapy for bleeding gastric variceal bleeding not amenable to IR guided therapies. ACG Case Re 2019; 6: 1-4 